\title{
Close Cathode Chamber: cost efficient and lightweight detector for tracking applications
}

\author{
Dezső Varga* \\ Wigner RCP, Budapest \\ E-mail: Dezso.Varga@cern.ch

\section{Gergő Hamar} \\ Wigner RCP, Budapest \\ E-mail: Hamar.Gergo@wigner.mta.hu
}

\begin{abstract}
The Close Cathode Chamber (CCC) is an asymmetric Multi-Wire Proportional Chamber (MWPC), which, owing to its specifically optimized field structure, has key advantages relative to the classical MWPC design. The CCC contains alternating field wires and anode (sense) wires, and the wire plane is asymmetric with respect to two parallel planes, being as close as $1.5 \mathrm{~mm}$ typically to one of the planes for $2 \mathrm{~mm}$ wire spacing. It is shown that this arrangement minimizes the dependence of the avalanche gain on detector wall planarity, and specifically, insensitivity to corresponding mechanical distortions or internal overpressure (causing e.g. bulging). Such feature allows one to build CCC chambers with small overall material budget, avoiding also the thick frames typical for MWPC-s. Careful studies confirmed that signal formation and position resolution correspond to that in classical MWPC-s. The dead zones created by internal support structures have been evaluated. CCC detectors have found application in a portable cosmic muon tracking system, proving their mechanical and operational stability under harsh and varying environmental conditions, and also in other hybrid detectors.
\end{abstract}

Technology and Instrumentation in Particle Physics 2014,

2-6 June, 2014

Amsterdam, the Netherlands

* Speaker. 


\section{Introduction}

The classical arrangement of Multi-Wire Proportional Chambers usually involve strong and heavy frames to hold the wire tension of the structure. The motivation of the studies presented below was to find an outline which is lightweight, easy to construct and tolerable to mechanical effects such as vibration, external tension or bulging by pressure. The concept called the "Close Cathode Chamber" [1] has proven to feature these properties, the most important one being the independence of the gain on cathode (baseplate) flatness. The key operational parameters and issues of large size construction [2] will be presented.

Early during the formulation of the concept, it has been noted that a design very similar to our final structure has been under investigation [3], as it is shown in Fig. 1. Both versions of asymmetric MWPC have alternate sense (anode) and field wires.
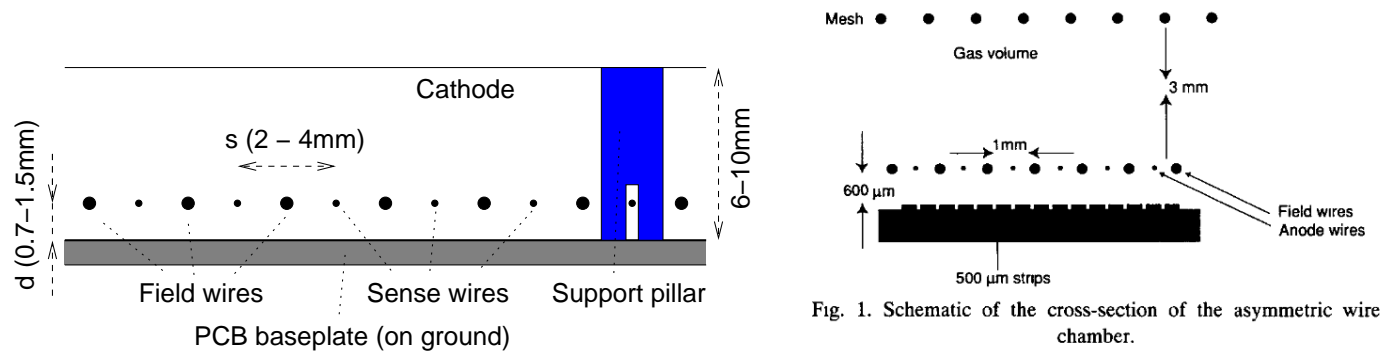

Fig. 1. Schematic of the cross-section of the asymmetric wire chamber.

Figure 1: Left panel: Outline of the CCC detector. The larger gap acts as charge collection (drift) region, whereas on the side of the lower, thinner gap the baseplate (on ground) may be segmented. Right panel: detector design from [3].

\section{The Close Cathode Chamber concept}

\subsection{Asymmetric MWPC prototype with inclined wire plane}

In order to study the effect of cathode flatness, a small prototype chamber has been constructed, with $21 \mu \mathrm{m}$ sense wires and $125 \mu \mathrm{m}$ field wires. The distance between sense wires is $4 \mathrm{~mm}$. As the chamber is asymmetric, the grounded plane will be referred to as "baseplate", whereas the "cathode" is the one defining the drift region, as shown on Fig. 1. In this prototype, the wire plane is inclined, above the baseplate by $1.5-2.0 \mathrm{~mm}$, as shown on Fig. 2 from the side. The upper cathode plane (on negative voltage $U_{C}$ ) is $10 \mathrm{~mm}$ from the lower cathode plane.

At a typical gain of around $10^{4}$ in the $\mathrm{Ar}+\mathrm{CO}_{2}$ 80:20 gas mixture, the field wire potential $U_{F}$ and the sense wire potential $U_{S}$ were around $-600 \mathrm{~V}$ and $+1000 \mathrm{~V}$ respectively.

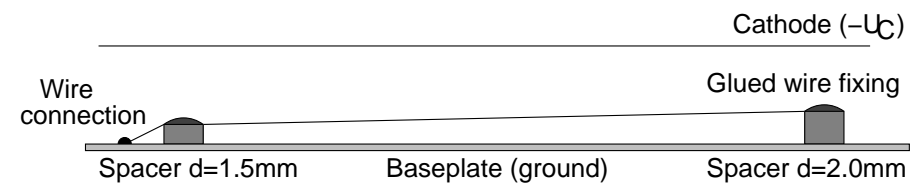

Figure 2: Side view of the prototype chamber with inclined wires 


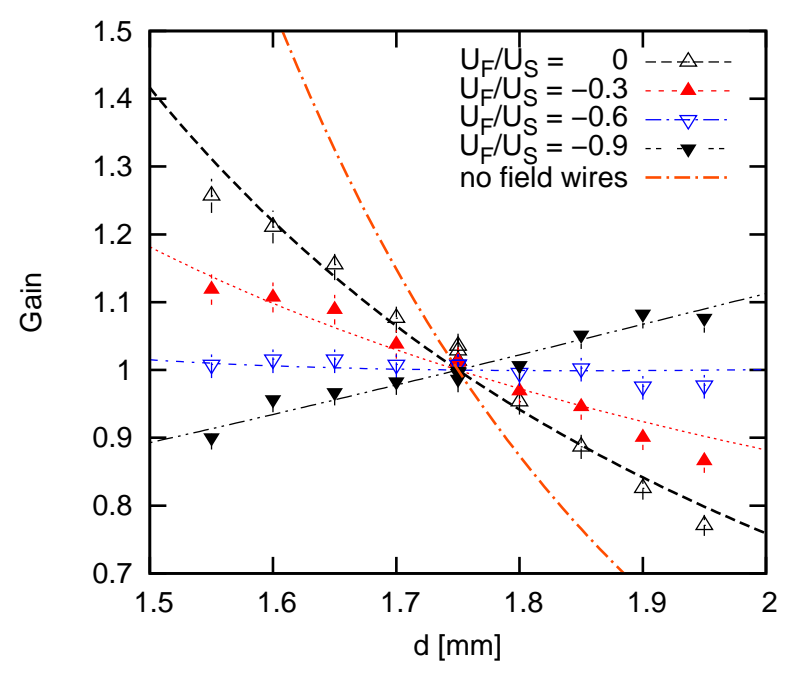

Figure 3: Relative gas amplification as a function of lower cathode and wire plane distance $d$, with different $U_{F} / U_{S}$ voltage ratios, at an approximately constant gain at the midpoint. The independence on $d$ is clearly demonstrated at $U_{F} / U_{S} \approx-0.6$.

Placing a radioactive beta source at any position, the amplification gain can be measured as a function of the wire plane distance from the cathode. Fig. 3 shows the measured gains, always normalized in a way such that the relative gain at $d=1.75 \mathrm{~mm}$ is unity. The measurement at $U_{F}=0$ is confirming the fact [4] that the gain increases with decreasing $d$ by a factor of two between $2 \mathrm{~mm}$ and $1.5 \mathrm{~mm}$.

If one choses negative voltage on $U_{F}$, the slope reduces, reaching practically $d$ independence at $U_{F} / U_{S}=-0.6$. At even larger negative $U_{F}$, the trend reverses, higher gain is achieved at larger $d$. The measurements thus demonstrate that in case of proper voltage choice the amplification gain can be independent of the cathode flatness in a very wide $d$ range. The effect is indeed dramatic if observed on a large prototype, shown in Fig. 4, with and without inner pressure, the former causing considerable chamber bulging.

The optimal ratio for $U_{F} / U_{S}$ does not depend on the gas gain or gas composition, being related to the static electric field inside the chamber; it does depend however on wire geometry and wire diameters. Qualitative explanation of the insensitivity [1] is based on the charge sharing between wires: if the whole wire plane moves further from the baseplate, then the charge reduces on the sense wires; whereas the sense wires are less shielded from the field wires (on opposite voltage) which acts towards increasing the sense wire charge. The two effects happen to compensate each other at the "optimal" voltage ratio.

\section{Features of the "Close Cathode" chamber setup}

\subsection{Electrostatic field in the chamber}

The calculated electric field lines in the chamber at optimal operation are shown in Fig. 5. The field lines are concentrated between the wires, and only modest field strength points towards the upper cathode or the baseplate. 

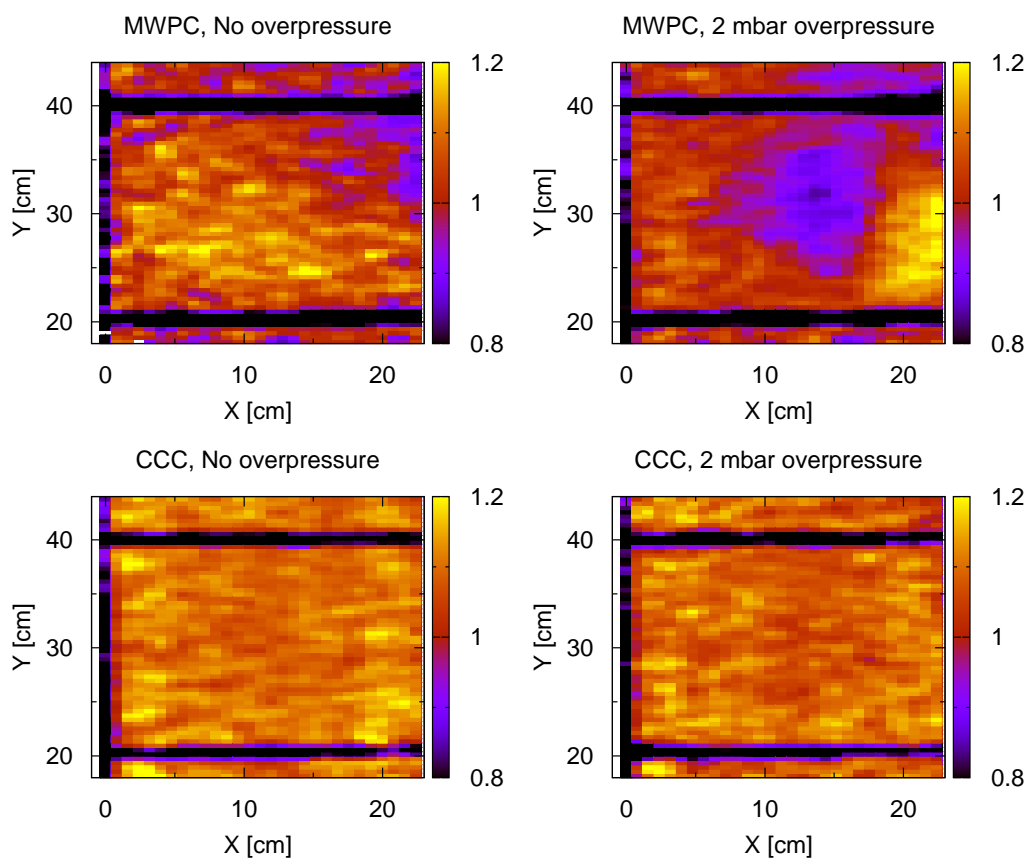

Figure 4: Gain measurement (relative, color coded) using cosmic muons over an approximately $20 \mathrm{~cm}$ by $20 \mathrm{~cm}$ region of the 1 meter chamber (region marked on Fig. 7). The left panels are without, the right panels are with 2 mbar internal overpressure. The top two panels were recorded with the "MWPC" voltage setting, whereas the bottom panels in the "CCC" mode. Note the reduced gain variations for the CCC arrangement.

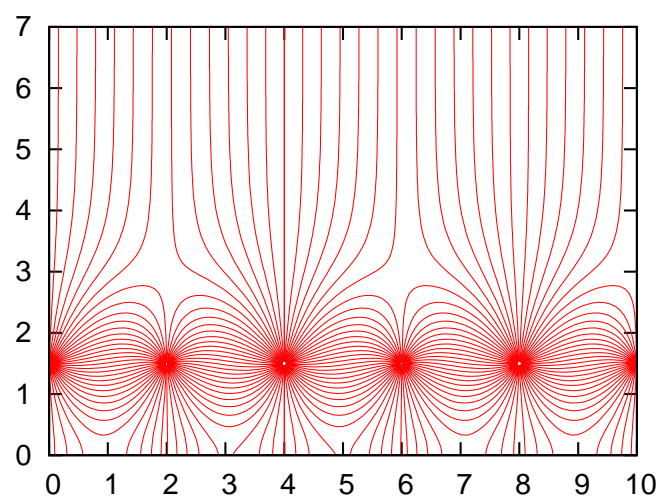

Figure 5: Field line distribution in the "Close Cathode" chamber prototype. Electrons are drifting from the upper charge collection zone towards the sense wires (positions $0,4,8$ ), whereas the electric field is reduced close to the bottom ground plane due to the concentration of the lines between the field- and sense-wires.

\subsubsection{Sensitivity to mechanical imperfections}

In the proposed "close cathode" setup, the requirement of the tolerance on $d$ is strongly relaxed. In the latest prototype optimized for this application, $d=1.5 \mathrm{~mm}$ was used with $4 \mathrm{~mm}$ sense wire spacing. Such a low $d$ value is generally difficult to achieve unless the cathode plane is kept flat within a few tens of microns. Prototypes with $d=0.7 \mathrm{~mm}$ were also produced and succesfully operated with $2 \mathrm{~mm}$ wire pitch. 

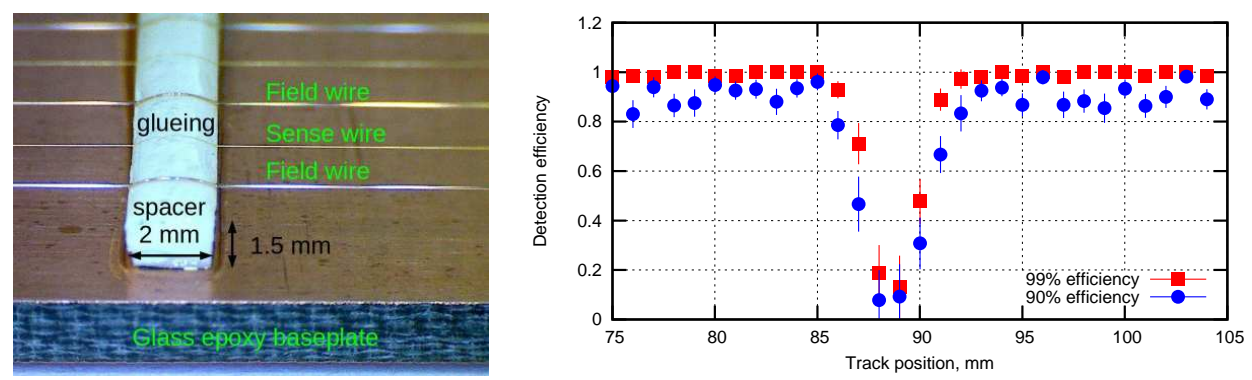

Figure 6: Left panel: Intermediate wire fixing spacer for reducing effective wire length. Right panel: Detection efficiency as a function of position in the chamber prototype, in the vincinity of the $2 \mathrm{~mm}$ wide intermediate spacer.

The other mechanical parameters of the chamber are easier to control during construction. Due to the fact that the field lines are concentrated between the wires, the precision of individual wire positions is critical, a fact which has been confirmed by detailed studies [1]: especially sense wire vertical (perpendicular to wire plane), and field wire horizontal (parallel to wire plane) positions are to be fixed precisely. Such wire positioning is however not difficult to achieve; in the prototypes, wires are glued to laser engraved plastic bars, thus ensuring both horizontal and vertical positioning.

\subsubsection{Signal formation}

The electric signal in an MWPC is initiated by the avalanche, and the signal shape is determined by the movement of the ions in the vicinity of the sense wire [4]. To study this, a simulation was implemented based on Garfield [5]. Individual electrons were followed by Monte-Carlo simulations in the appropriate $\mathrm{Ar}-\mathrm{CO}_{2}$ gas mixture.

One can conclude, that in line with the intuitive expectation, the signal on the closer cathode has the same time dependence for classical MPWC-s and CCC, since the ions see similar field lines around the sense wires. The studies confirm the qualitative experimental finding, that the Close Cathode Chamber structure behaves as a classical MWPC from the point of view of signal formation, and therefore all information gathered with MWPC-s is applicable for that case too.

\section{Detector construction}

The general structure of MWPCs contains either a solid cathode plane, or a solid frame to hold wire tension. The cathode flatness needs to be maintained in spite of the bending force exerted by wire tension, and of pressure change inside the chamber. In the "close cathode" setup, precision requirement on flatness is largely reduced, therefore thinner cathode plane and baseplate may be used. In addition, the bending effect of the wires on the baseplate is smaller (due to the smaller $d$ ).

In case of larger chambers the electrostatic force among wires may cause an instability. This may be solved by a thin intermediate spacer which holds no wire tension but keeps the wires in place in all directions (see Fig. 6). The width of the "dead" area caused by the spacer was studied using perpendicularly incident cosmic muons in one of the prototypes, to ensure that it does not create large acceptance holes in the complete detector. The local efficiency loss depends on the 
mean efficiency of the chamber. The measurement has been done at two mean efficiencies, $90 \%$ and $99 \%$, as shown on the right panel in Fig. 6 .
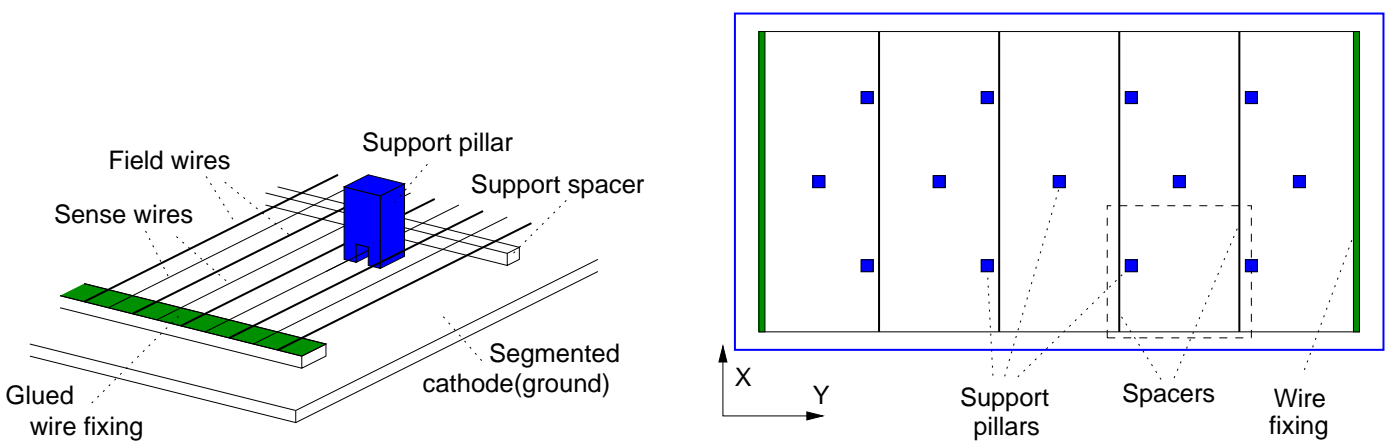

Figure 7: Left panel: Mechanical structure inside the CCC chamber. Wire fixations hold the wires in place, while wire supports and pillars should be introduced for large area detectors. Right panel: Schematic top view of the arrangement of the pillars and the spacers on the whole surface of the large prototype chamber.

The physical width of the spacer was $2 \mathrm{~mm}$, and the broadening of measured inefficiency is around $4 \mathrm{~mm}$, which means that in a distance in the order of $1 \mathrm{~mm}$ from the spacer, the gain is recovered to a level sufficient for detection. The outline of the mechanical structure including wire fixation, a wire support and a pillar is shown in Fig. 7.

The possibility of "large size" construction was tested on a chamber which had $0.5 \mathrm{~m}$ width and $1 \mathrm{~m}$ length, with wires stretched along the longer side. The wire support and pillar structure is also shown in Fig. 7. The test unit has been built with $0.5 \mathrm{~mm}$ thick G10 cathode and $1 \mathrm{~mm}$ G10 baseplate. Even though the total thickness of such a chamber was closely $12 \mathrm{~mm}$, the structure was rather rigid, holding its own weight safely. The total weight (half square meter) was $2 \mathrm{~kg}$.

\section{Applications}

The CCC has found various applications which exploited the advantages of the concept, namely tolerance of mechanical disturbances and ease of construction. The feature of low material budget was especially useful as a proposed trigger system for the proposed ALICE VHMPID detector [6].

\subsection{Cosmic muon tracking}

A cosmic muon tracking station has been consructed from CCC layers, each with two dimensional readout. The detector was operating independently for weeks at a depth of about $50 \mathrm{~m}$ in a natural cave (see Fig. 8, mapping the cosmic muon flux, and hence the soil density above the measurement point [7].

\subsection{TCPD: Thick GEM + CCC hybrid}

The CCC detector may be used in combination with a Thick GEM [8], a hybrid which features the advantages of both techniques: high UV photon sensitivity of the TGEM and inherent stability of the CCC. The system has been successfully operated and shown high senstivity to UV photons generated by Cherenkov radiation, shown in Fig. 9. 

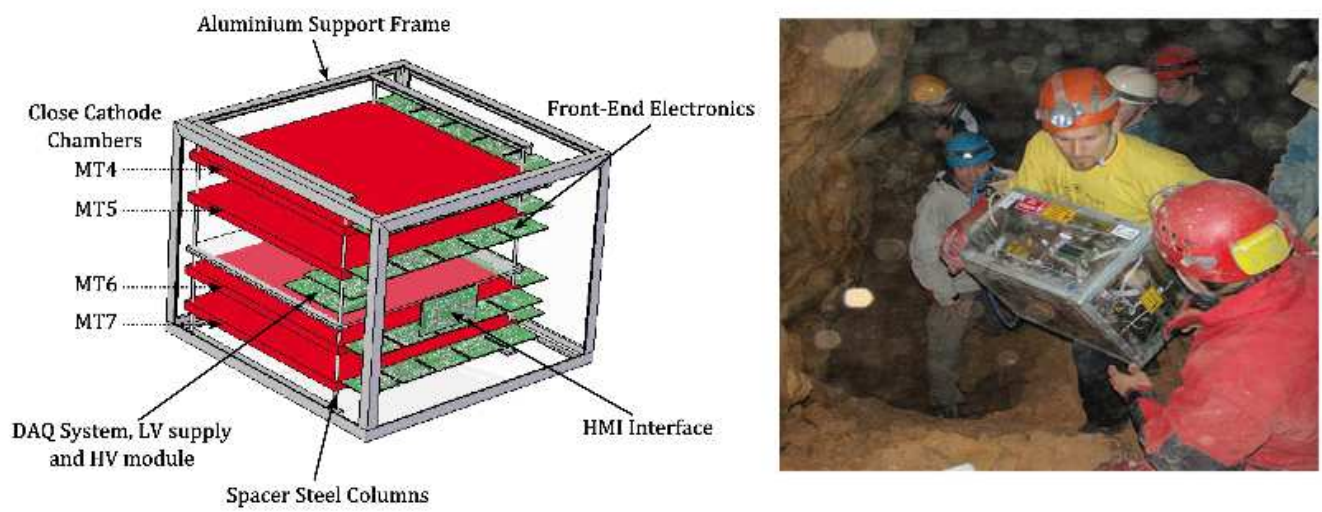

Figure 8: Underground detector constructed from CCC tracking layers (left) and deployment to a recently discovered natural cave (right)
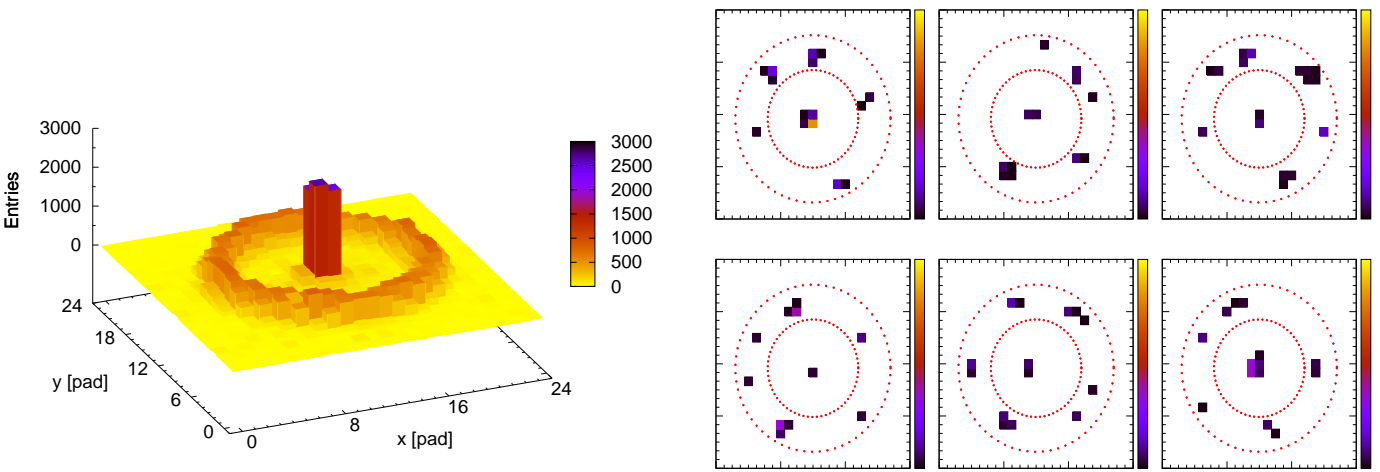

Figure 9: Left panel: Cherenkov photons accumulated in a TCPD detector. Right panel: Single events showing identifiable ring patterns

\subsection{Leopard high resolution scanning}

The TCPD, coupled to a high resolution UV source allows one to create high resolution map of single photo-electron efficiency and gain of the TGEM layers [9]. In fact, the CCC itself can be made sensitive to single photo-electrons at a typical gain of $10^{5}$, see Fig. 10, which gives a useful tool for the CCC gain calibration in the TGEM+CCC hybrid.

\section{Conclusions}

The Close Cathode Chamber detector, an innovative MWPC features advantages with respect to the classical design of MWPC-s, especially in tolerance of mechanical disturbances and low material budget. Various applications have emerged in which CCC chambers can be used as tracking detectors, or part of hybrids coupled to an MPGD stage.

\section{Acknowledgements}

We would like to thank the assistance form the members of the REGARD group (Wigner 


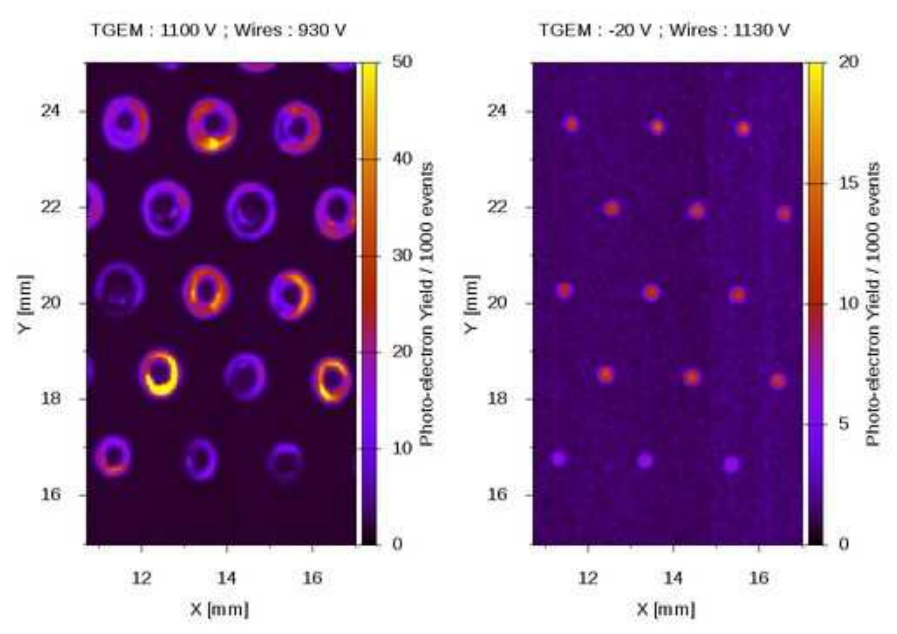

Figure 10: Image taken with the Leopard high resolution scanning system. The left image maps the photon sensitivity of a TGEM, whereas on the right, the photons which pass through the holes, and thus measured only by the CCC, are still well detectable.

RCP, Budapest). This work has been supported by the "Lendület" Programme of the Hungarian Academy of Sciences (LP2013-60).

\section{References}

[1] D.Varga, G.Hamar, G.Kiss, Nucl.Instr.Meth A 648 (2011) 163-167

[2] D.Varga, G. Kiss, G.Hamar, Gy.Bencédi, Nucl.Instr.Meth A 698 (2013) 11-18

[3] G. Charpak, I. Crotty, Y. Giomataris, L. Ropelewski and M. C. S. Williams Nucl. Instrum. Meth. A 346 (1994) 506-509.

[4] W.Blum, L.Rolandi, Particle Detection with Drift Chambers, Springer-Verlag (1993)

[5] http://garfield.web.cern.ch/garfield/

[6] A.Di Mauro et al, Nucl.Instr.Meth. A 639 (2011) 274-277

[7] G. G. Barnaföldi, et al, Nucl. Instr. Meth. A 689 (2012) 60-69

[8] G. Hamar, D. Varga, Journal of Inst. 8 (2013) C12038

[9] G. Hamar, D. Varga. Nucl. Instr. Meth. A 694 (2012) 16-23 